\title{
Spin and pseudospin symmetry along with orbital dependency of the Dirac-Hulthén problem
}

\author{
Sameer M. Ikhdair, ${ }^{1, *}$ Cüneyt Berkdemir, ${ }^{2,+t}$ and Ramazan Sever ${ }^{3, t}$ \\ ${ }^{1}$ Physics Department, Near East University, Nicosia, North Cyprus, Turkey \\ ${ }^{2}$ Physics Department, Erciyes University, 38039 Kayseri, Turkey \\ ${ }^{3}$ Physics Department, Middle East Technical University, 06531 Ankara, Turkey
}

(Dated: October 8, 2018)

\begin{abstract}
The role of the Hulthén potential on the spin and pseudospin symmetry solutions is investigated systematically by solving the Dirac equation with attractive scalar $S(\vec{r})$ and repulsive vector $V(\vec{r})$ potentials. The spin and pseudospin symmetry along with orbital dependency (pseudospin-orbit and spin-orbit dependent couplings) of the Dirac equation are included to the solution by introducing the Hulthén-square approximation. This effective approach is based on forming the spin and pseudo-centrifugal kinetic energy term from the square of the Hulthén potential. The analytical solutions of the Dirac equation for the Hulthén potential with the spin-orbit and pseudospinorbit-dependent couplings are obtained by using the Nikiforov-Uvarov (NU) method. The energy eigenvalue equations and wave functions for various degenerate states are presented for several spin-orbital, pseudospin-orbital and radial quantum numbers under the condition of the spin and pseudospin symmetry.
\end{abstract}

Keywords: Spin and pseudospin symmetry; orbital dependency; Dirac equation; Hulthén potential; Nikiforov-Uvarov Method.

PACS numbers: 03.65.Ge; 03.65.Pm; 11.30.Pb; 21.60.Cs; 31.30.Jv

\footnotetext{
*E-mail: sikhdair@neu.edu.tr

${ }^{\dagger}$ E-mail: berkdemir@erciyes.edu.tr

${ }_{\ddagger}^{\ddagger}$ E-mail: sever@metu.edu.tr
} 


\section{INTRODUCTION}

The spin and pseudospin symmetry [1,2] observed originally almost 40 years ago as a mechanism to explain different aspects of the nuclear structure is one of the most interesting phenomena in the relativistic quantum mechanics. It plays a crucial role for a Dirac hamiltonian with realistic scalar $S(\vec{r})$ and vector $V(\vec{r})$ potentials, for nucleon spectrum in nuclei, for the existence of identical bands in superdeformed nuclei, etc [3]. The key feature of the pseudospin symmetry is based on the small energy difference between single-nucleon doublets with quantum numbers $n_{r}, \ell, j=\ell+1 / 2$ and $n_{r}-1, \ell+2, j=\ell+3 / 2$, where $n_{r}, \ell$ and $j$ are the single nucleon radial, orbital and total angular quantum numbers, respectively. These quantum numbers are relabelled as pseudospin doublets; $\ell+1=\tilde{\ell}$ is the "pseudo" orbital angular momentum, $\tilde{s}=1 / 2$ is the "pseudo" spin and $j=\tilde{\ell} \pm \tilde{s}$ is the total "pseudo" angular momentum for the two states in the doublet [4]. For example, " $n_{r} s_{1 / 2},\left(n_{r}-1\right) d_{3 / 2} "$ is valid for $\tilde{\ell}=1, " n_{r} p_{3 / 2},\left(n_{r}-1\right) f_{5 / 2}$ is valid for $\tilde{\ell}=2$, etc. Another key feature is the single-particle Hamiltonian of the oscillator shell model. This means that the pseudospin concept in the nuclear theory is a division of the single-particle total angular momentum into pseudo rather than normal orbital and spin parts. The shell model implies that nucleons move in a relativistic mean field produced by the interactions between nucleons. The relativistic dynamics of nucleons moving in the relativistic mean field are described by using the Dirac equation and not the Schrödinger equation.

The pseudospin symmetry concept is investigated by the framework of the Dirac equation and occurs as a symmetry of the Dirac hamiltonian when an attractive scalar $S(\vec{r})$ and a repulsive vector $V(\vec{r})$ potentials near equal to each other in magnitude, but opposite in sign, i.e., $S(\vec{r}) \sim-V(\vec{r})$. On the other hand, the sum of the vector and scalar potentials in the Dirac equation is a constant, i.e., $V(\vec{r})+S(\vec{r})=$ constant, for the solution of the pseudospin symmetry in nuclei. This condition has been found by Ginocchio [5] and applied to the case of the spherical harmonic oscillator [6]. Meng et al [7] showed that the pseudospin symmetry is exact under the condition of $d(V(\vec{r})+S(\vec{r})) / d r=0$. Lisboa et al. studied the generalized harmonic oscillator for spin-1/2 particles by setting either $\Sigma(\vec{r})=V(\vec{r})+S(\vec{r})=0$ or $\Delta(\vec{r})=V(\vec{r})-S(\vec{r})=0[8]$. A necessary condition for occurrence of the pseudospin symmetry in nuclei is to consider the case $\Sigma(\vec{r})=0[3,5,9,10]$. For more realistic nuclear systems, the quality of the pseudospin symmetry is increased in the framework of the single- 
particle relativistic models and hence the competition between the pseudo-centrifugal barrier and the pseudospin-orbital potential is completed in the onset of pseudospin symmetry [11]. The Dirac equation with the pseudospin symmetry is solved numerically for nucleons which move independently in the relativistic mean field with external scalar and vector potentials $[12,13]$. In addition to the numerical solutions, some analytical solutions are also discussed for solving the Dirac equation for some realistic potentials [14-17] with the pseudospin symmetry. The analytical solutions show that under the condition of pseudospin symmetry, the exact solution of the Dirac equation gives the bound-state energy spectra and spinor wave functions [18-20].

The aim of this paper is to present an analytical bound state solutions of the Dirac equation for the Hulthén potential under the conditions of the exact pseudospin symmetry and exact spin symmetry. To obtain a general solution for all values of the pseudospin (spin) quantum numbers, the pseudospin (spin) symmetry and orbital dependency, pseudospin-orbit (spin-orbit) dependent coupling are included to the lower component of the Dirac equation as an integer quantum number. This component has the structure of the Schrödinger-like equations with the pseudo-centrifugal (spin-centrifugal) kinetic energy term and its solution is analyzed by using some algebraic methods and effective approaches. One of these effective approaches is applied to the pseudo-centrifugal (spin-symmetry) kinetic energy term in the case of $\tilde{\ell}>0(\ell>0)$ and also an effective potential suggested in the form of the square of the Hulthén potential is taken into account instead of the pseudo-centrifugal kinetic energy term. For small values of the radial coordinate $r$, this effective potential gives a centrifugal energy term in the first approximation. Therefore, the pseudo-centrifugal (spin-centrifugal) kinetic energy term is accepted as an effective term in this region. It is worthy to state that Jia et al $[21,22]$ have proposed an improved new approximation scheme to deal with the centrifugal kinetic energy term in the solution of the Schrödinger-Hulthén problem. Using this approximation scheme, Jia et al $[23,24]$ have obtained approximate analytical solutions for the Dirac-generalized Pöschl-Teller and Klein-Gordon-Pöschl-Teller problems including the centrifugal kinetic energy term. Recently, Ikhdair [25] has applied the approximation scheme to deal with the orbital centrifugal term in the SchrödingerManning-Rosen problem using the Nikiforov-Uvarov method. Further, the approximation has also been applied to the Schrödinger-Hulthén problem using the improved quantization rule $[26]$. 
In the present work, the Dirac equation for the Hulthén potential is arranged under the condition of the exact pseudospin (spin) symmetry and it's solution is obtained systematically by using the Nikiforov-Uvarov (NU) method [27-31]. As an application of the Dirac-Hulthén problem with the pseudospin (spin) symmetry, the relativistic eigenvalue spectrum for various degenerate states is presented for several pseudo-orbital (spin-orbital) and pseudospin (spin) quantum numbers.

The structure of the paper is as follows. In Sec. 2, the basic ideas of the Nikiforov-Uvarov (NU) method are outlined in short. In Sec. 3, the Dirac equation is briefly introduced for the spin and pseudospin symmetry solutions. In Sec. 4, the Hulthén potential is substituted into the lower component of the Dirac equation and the pseudo-centrifugal (or spin-centrifugal) kinetic energy term is replaced by the square of the Hulthén potential to apply the Hulthén square approximation. The main results obtained in previous sections are connected by means of the main equation of the NU method. Lastly, the general procedures of the solution method are followed to obtain the energy eigenvalue equation and two-spinor wave functions. Results and conclusions are performed in Sec. 5 .

\section{BASIC IDEAS OF THE NIKIFOROV-UVAROV (NU) METHOD}

It is especially well known that the solutions of the Schrödinger and Schrödinger-like equations including the centrifugal barrier and/or the spin-orbit coupling terms have not been obtained straightforwardly for the exponential-type potentials such as Morse, Hulthén, Woods-Saxon, etc [32]. Although the exact solution of the Schrödinger equation for the exponential-type potentials has been obtained for $\ell=0$, any $\ell$-state solutions have been given approximately by using some analytical methods under a certain number of restrictions $[33,34]$. One of the calculational tools utilized in these studies is the NU method. This technique is based on solving the hypergeometric type second-order differential equations by means of the special orthogonal functions [35]. For a given potential, the Schrödinger or Schrödinger-like equations in spherical coordinates are reduced to a generalized equation of hypergeometric type with an appropriate coordinate transformation $r \rightarrow s$ and then they are solved systematically to find the exact or particular solutions. The main equation which 
is closely associated with the method is given in the following form [27]

$$
\psi^{\prime \prime}(s)+\frac{\widetilde{\tau}(s)}{\sigma(s)} \psi^{\prime}(s)+\frac{\widetilde{\sigma}(s)}{\sigma^{2}(s)} \psi(s)=0
$$

where $\sigma(s)$ and $\widetilde{\sigma}(s)$ are polynomials at most second-degree, $\widetilde{\tau}(s)$ is a first-degree polynomial and $\psi(s)$ is a function of the hypergeometric type.

Let us now try to reduce Eq.(1) to a comprehensible form by taking $\psi(s)=\phi(s) y(s)$ and choosing an appropriate function $\phi(s)$ :

$$
y^{\prime \prime}(s)+\left(2 \frac{\phi^{\prime}(s)}{\phi(s)}+\frac{\widetilde{\tau}(s)}{\sigma(s)}\right) y^{\prime}(s)+\left(\frac{\phi^{\prime \prime}(s)}{\phi(s)}+\frac{\phi^{\prime}(s)}{\phi(s)} \frac{\widetilde{\tau}(s)}{\sigma(s)}+\frac{\widetilde{\sigma}(s)}{\sigma^{2}(s)}\right) y(s)=0 .
$$

At the first stage, Eq.(2) can be seen to be more complicated than the main equation, Eq.(1). To ensure the reasonable understanding, the coefficient of $y^{\prime}(s)$ is taken in the form $\tau(s) / \sigma(s)$, where $\tau(s)$ is a polynomial of degree at most one, i.e.,

$$
2 \frac{\phi^{\prime}(s)}{\phi(s)}+\frac{\widetilde{\tau}(s)}{\sigma(s)}=\frac{\tau(s)}{\sigma(s)}
$$

and hence the most regular form is obtained as follows,

$$
\frac{\phi^{\prime}(s)}{\phi(s)}=\frac{\pi(s)}{\sigma(s)}
$$

where

$$
\pi(s)=\frac{1}{2}[\tau(s)-\widetilde{\tau}(s)] .
$$

The most useful demonstration of Eq. (5) is

$$
\tau(s)=\widetilde{\tau}(s)+2 \pi(s)
$$

The new parameter $\pi(s)$ is a polynomial of degree at most one. In addition, the term $\phi^{\prime \prime}(s) / \phi(s)$ which appears in the coefficient of $y(s)$ in Eq.(2) is arranged as follows

$$
\frac{\phi^{\prime \prime}(s)}{\phi(s)}=\left(\frac{\phi^{\prime}(s)}{\phi(s)}\right)^{\prime}+\left(\frac{\phi^{\prime}(s)}{\phi(s)}\right)^{2}=\left(\frac{\pi(s)}{\sigma(s)}\right)^{\prime}+\left(\frac{\pi(s)}{\sigma(s)}\right)^{2} .
$$

In this case, the coefficient of $y(s)$ is transformed into a more suitable arrangement by taking the form in Eq.(4);

$$
\frac{\phi^{\prime \prime}(s)}{\phi(s)}+\frac{\phi^{\prime}(s)}{\phi(s)} \frac{\widetilde{\tau}(s)}{\sigma(s)}+\frac{\tilde{\sigma}(s)}{\sigma^{2}(s)}=\frac{\bar{\sigma}(s)}{\sigma^{2}(s)}
$$

where

$$
\bar{\sigma}(s)=\widetilde{\sigma}(s)+\pi^{2}(s)+\pi(s)\left[\widetilde{\tau}(s)-\sigma^{\prime}(s)\right]+\pi^{\prime}(s) \sigma(s) .
$$


Substituting the right-hand sides of Eq.(3) and Eq.(8) into Eq.(2), an equation of the same type as Eq.(1) is obtained as

$$
y^{\prime \prime}(s)+\frac{\tau(s)}{\sigma(s)} y^{\prime}(s)+\frac{\bar{\sigma}(s)}{\sigma^{2}(s)} y(s)=0 .
$$

As a consequence of the above algebraic transformations, the functional form of Eq.(1) is protected by following a systematic way. Therefore, the transformations allow us to replace the function of the hypergeometric type $\psi(s)$ by the substitution $\phi(s) y(s)$, where $\phi(s)$ satisfies Eq.(4) whit an arbitrary linear polynomial $\pi(s)$. If the polynomial $\bar{\sigma}(s)$ in Eq.(10) is divisible by $\sigma(s)$, i.e.,

$$
\bar{\sigma}(s)=\lambda \sigma(s)
$$

where $\lambda$ is a constant, Eq.(10) is reduced to an equation of hypergeometric type

$$
\sigma(s) y^{\prime \prime}+\tau(s) y^{\prime}+\lambda y=0
$$

and also its solution is given as a function of hypergeometric type [35]. To determine the polynomial $\pi(s)$, Eq.(9) is compared with Eq.(11) and then a quadratic equation for $\pi(s)$ is obtained as follows,

$$
\pi^{2}(s)+\pi(s)\left[\widetilde{\tau}(s)-\sigma^{\prime}(s)\right]+\widetilde{\sigma}(s)-k \sigma(s),
$$

where

$$
k=\lambda-\pi^{\prime}(s)
$$

The solution of this quadratic equation for $\pi(s)$ yields the following equality

$$
\pi(s)=\frac{\sigma^{\prime}(s)-\widetilde{\tau}(s)}{2} \pm \sqrt{\left(\frac{\sigma^{\prime}(s)-\widetilde{\tau}(s)}{2}\right)^{2}-\widetilde{\sigma}(s)+k \sigma(s)}
$$

In order to obtain the possible solutions according to the plus and minus signs of Eq.(15), the parameter $k$ within the square root sign must be known explicitly. To provide this requirement, the expression under the square root sign has to be the square of a polynomial, since $\pi(s)$ is a polynomial of degree at most one. In this case, an equation of the quadratic form is available for the constant $k$. Setting the discriminant of this quadratic equal to zero, the constant $k$ is determined clearly. After determining $k$, the polynomial $\pi(s)$ is obtained from Eq.(15), and then $\tau(s)$ and $\lambda$ are also obtained by using Eq.(5) and Eq.(14), respectively. 
A common trend which is followed to generalize the solutions of Eq.(12) is to show that all the derivatives of functions of hypergeometric type are also of hypergeometric type. For this purpose, Eq.(12) is differentiated by using the representation $v_{1}(s)=y^{\prime}(s)$

$$
\sigma(s) v_{1}^{\prime \prime}(s)+\tau_{1}(s) v_{1}^{\prime}(s)+\mu_{1} v_{1}(s)=0
$$

where $\tau_{1}(s)=\tau(s)+\sigma^{\prime}(s)$ and $\mu_{1}=\lambda+\tau^{\prime}(s)$. $\tau_{1}(s)$ is a polynomial of degree at most one and $\mu_{1}$ is independent of the variable $s$. It is clear that Eq.(16) is an equation of hypergeometric type again. By taking $v_{2}(s)=y^{\prime \prime}(s)$ as a new representation, the second derivation of Eq.(12) becomes

$$
\sigma(s) v_{2}^{\prime \prime}(s)+\tau_{2}(s) v_{2}^{\prime}(s)+\mu_{2} v_{2}(s)=0
$$

where

$$
\begin{aligned}
& \tau_{2}(s)=\tau_{1}(s)+\sigma^{\prime}(s)=\tau(s)+2 \sigma^{\prime}(s), \\
& \mu_{2}=\mu_{1}+\tau_{1}^{\prime}(s)=\lambda+2 \tau^{\prime}(s)+\sigma^{\prime \prime}(s) .
\end{aligned}
$$

In a similar way, an equation of hypergeometric type for $v_{n}(s)=y^{(n)}(s)$ is constructed as a family of particular solutions of Eq.(12) corresponding to a given $\lambda$;

$$
\sigma(s) v_{n}^{\prime \prime}(s)+\tau_{n}(s) v_{n}^{\prime}(s)+\mu_{n} v_{n}(s)=0
$$

and here the general recurrence relations for $\tau_{n}(s)$ and $\mu_{n}$ are found as follows, respectively,

$$
\begin{gathered}
\tau_{n}(s)=\tau(s)+n \sigma^{\prime}(s), \\
\mu_{n}=\lambda+n \tau^{\prime}(s)+\frac{n(n-1)}{2} \sigma^{\prime \prime}(s) .
\end{gathered}
$$

When $\mu_{n}=0$, Eq.(22) becomes as follows

$$
\lambda=\lambda_{n}=-n \tau^{\prime}(s)-\frac{n(n-1)}{2} \sigma^{\prime \prime}(s), \quad(n=0,1,2, \ldots)
$$

and then Eq.(20) has a particular solution of the form

$$
y(s)=y_{n}(s)=\frac{B_{n}}{\rho(s)} \frac{d^{n}}{d r^{n}}\left[\sigma^{n}(s) \rho(s)\right],
$$

which is the Rodrigues relation of degree $n$ and $\rho(s)$ is the weight function satisfying the differential equation

$$
[\sigma(r) \rho(r)]^{\prime}=\tau(r) \rho(r)
$$

To obtain an eigenvalue solution through the NU method, the relationship between $\lambda$ and $\lambda_{n}$ must be set up by means of Eq.(14) and Eq.(23). 


\section{DIRAC EQUATION}

In the relativistic description, the Dirac equation of a single-nucleon with the mass $\mu$ moving in an attractive scalar potential $S(\vec{r})$ and a repulsive vector potential $V(\vec{r})$ can be written as

$$
\left[\vec{\alpha} \cdot c \vec{P}+\beta\left(\mu c^{2}+S(\vec{r})\right)+V(\vec{r})\right] \psi_{n_{r} \kappa}(\vec{r})=E_{n_{r} \kappa} \psi_{n_{r} \kappa}(\vec{r}),
$$

where

$$
\vec{P}=-i \hbar \vec{\nabla}, \quad \vec{\alpha}=\left(\begin{array}{cc}
0 & \vec{\sigma} \\
\vec{\sigma} & 0
\end{array}\right), \quad \beta=\left(\begin{array}{cc}
0 & I \\
-I & 0
\end{array}\right),
$$

with $\vec{\sigma}$ is the vector Pauli spin matrix and $I$ is the identity matrix. $\vec{P}$ is the three momentum operators, $\vec{\alpha}$ and $\beta$ are the usual $4 \times 4$ Dirac matrices [36], $c$ is the velocity of light in vacuum and $\hbar$ is the Planck's constant divided by $2 \pi . E_{n_{r} \kappa}$ denotes the relativistic energy eigenvalues of the Dirac particle. For nuclei with spherical symmetry, $S(\vec{r})$ and $V(\vec{r})$ potentials in Eq. $(24)$ represent only the radial coordinates, i.e., $S(\vec{r})=S(r)$ and $V(\vec{r})=V(r)$, where $r$ is the magnitude of $\vec{r}$. The spinor wave functions $\psi_{n_{r} \kappa}(\vec{r})$ can be written in the following form

$$
\psi_{n_{r} \kappa}(\vec{r})=\frac{1}{r}\left(\begin{array}{c}
F_{n_{r} \kappa}(r)\left[Y_{\ell}(\theta, \phi) \chi_{ \pm}\right]_{m}^{(j)} \\
i G_{n_{r} \kappa}(r)\left[Y_{\tilde{\ell}}(\theta, \phi) \chi_{ \pm}\right]_{m}^{(j)}
\end{array}\right)
$$

where $Y_{\ell}(\theta, \phi)\left(Y_{\tilde{\ell}}(\theta, \phi)\right)$ and $\chi_{ \pm}$are the spin (pseudospin) spherical harmonic and spin wave function which are coupled to angular momentum $j$ with projection $m$, respectively. $F_{n_{r} \kappa}(r)$ and $G_{n_{r} \kappa}(r)$ are the radial wave functions for the upper and lower components, respectively. The label $\kappa$ has two explanations; the aligned spin $j=\ell+1 / 2\left(s_{1 / 2}, p_{3 / 2}\right.$, etc. $)$ is valid for the case of $\kappa=-(j+1 / 2)$ and then $\tilde{\ell}=\ell+1$, while the unaligned spin $j=\ell-1 / 2\left(p_{1 / 2}, d_{3 / 2}\right.$, etc. $)$ is valid for the case of $\kappa=(j+1 / 2)$ and then $\tilde{\ell}=\ell-1$. Thus, the quantum number $\kappa$ and the radial quantum number $n_{r}$ are sufficient to label the Dirac eigenstates. The Dirac equation given in Eq.(24) may be reduced to a set of two coupled ordinary differential equations (in units of $c=\hbar=1)$ :

$$
\begin{aligned}
& \left(\frac{d}{d r}+\frac{\kappa}{r}\right) F_{n_{r} \kappa}(r)=\left(\mu+E_{n_{r} \kappa}-\Delta(r)\right) G_{n_{r} \kappa}(r), \\
& \left(\frac{d}{d r}-\frac{\kappa}{r}\right) G_{n_{r} \kappa}(r)=\left(\mu-E_{n_{r} \kappa}+\Sigma(r)\right) F_{n_{r} \kappa}(r),
\end{aligned}
$$


where $\Delta(r)=V(r)-S(r)$ and $\Sigma(r)=V(r)+S(r)$ are the difference and the sum potentials, respectively. By substituting

$$
F_{n_{r} \kappa}(r)=\frac{1}{\left(\mu-E_{n_{r} \kappa}+\Sigma(r)\right)}\left(\frac{d}{d r}-\frac{\kappa}{r}\right) G_{n_{r} \kappa}(r),
$$

into Eq.(27), the following second order Schrödinger-like differential equation for $G_{n_{r} \kappa}(r)$ can be obtained as

$$
\left(\frac{d^{2}}{d r^{2}}-\frac{\kappa(\kappa-1)}{r^{2}}-\left(\mu+E_{n_{r} \kappa}-\Delta(r)\right)\left(\mu-E_{n_{r} \kappa}+\Sigma(r)\right)-\frac{\frac{d \Sigma}{d r}\left(\frac{d}{d r}-\frac{\kappa}{r}\right)}{\mu-E_{n_{r} \kappa}+\Sigma(r)}\right) G_{n_{r} \kappa}(r)=0
$$

where $E_{n_{r} \kappa} \neq \mu$ when $\Sigma(r)=0$ (exact pseudospin symmetry). Further, a similar equation for $F_{n_{r} \kappa}(r)$ can be obtained as follows

$$
\left(\frac{d^{2}}{d r^{2}}-\frac{\kappa(\kappa+1)}{r^{2}}-\left(\mu+E_{n_{r} \kappa}-\Delta(r)\right)\left(\mu-E_{n_{r} \kappa}+\Sigma(r)\right)+\frac{\frac{d \Delta}{d r}\left(\frac{d}{d r}+\frac{\kappa}{r}\right)}{\mu+E_{n_{r} \kappa}-\Delta(r)}\right) F_{n_{r} \kappa}(r)=0,
$$

where $E_{n_{r} \kappa} \neq-\mu$ when $\Delta(r)=0$ (exact spin symmetry). Under the condition of exact spin symmetry, $(d \Delta(r) / d r=0$, i.e., $\Delta(r)=C=$ constant), Eq. (30) turns out to be

$$
\left(\frac{d^{2}}{d r^{2}}-\frac{\ell(\ell+1)}{r^{2}}-\left(\mu+E_{n_{r} \kappa}-C\right) \Sigma(r)+E_{n_{r} \kappa}^{2}-\mu^{2}+C\left(\mu-E_{n_{r} \kappa}\right)\right) F_{n_{r} \kappa}(r)=0,
$$

where $\ell(\ell+1)$ comes from $\kappa(\kappa+1)$ and $\ell(\ell+1) / r^{2}$ is the spin-centrifugal kinetic energy term. On the other hand, under the condition of the exact pseudospin symmetry $(d \Sigma(r) / d r=0$, i.e., $\Sigma(r)=C=$ constant $)$, Eq. (29) is reduced to the form

$$
\left(\frac{d^{2}}{d r^{2}}-\frac{\tilde{\ell}(\tilde{\ell}+1)}{r^{2}}+\left(\mu-E_{n_{r} \kappa}+C\right) \Delta(r)+E_{n_{r} \kappa}^{2}-\mu^{2}-C\left(\mu+E_{n_{r} \kappa}\right)\right) G_{n_{r} \kappa}(r)=0
$$

where $\tilde{\ell}(\tilde{\ell}+1)$ comes from $\kappa(\kappa-1)$ and $\tilde{\ell}(\tilde{\ell}+1) / r^{2}$ is the pseudo-centrifugal kinetic energy term. According to the original definition of the pseudo-orbital angular momentum, the cases $\tilde{\ell}=\kappa-1$ and $\tilde{\ell}=-\kappa$ are valid for $\kappa>0$ and $\kappa<0$, respectively. Therefore, the degenerate states come into existence with the same $\tilde{\ell}$ but different $\kappa$, generating pseudospin symmetry. Another important point which is necessary to be said on Eq.(32) is that the radial part of the spinor wave function $\psi_{n_{r} \kappa}(\vec{r})$ must satisfy the boundary conditions that $G_{n_{r} \kappa}(r) / r$ becomes zero when $r \rightarrow \infty$, and $G_{n_{r} \kappa}(r) / r$ is finite at $r=0$. 


\section{BOUND STATE SOLUTION BY MEANS OF THE NU METHOD}

\section{A. Hulthén Square Approximation}

In this section, we shall involve the Hulthén potential to solve the Dirac equation given in Eq.(32), meaning that the potential $\Delta(r)$ is exponential in $r$ and the pseudo-centrifugal kinetic energy term is quadratic in $1 / r$. The exponential potential in $r$ is the famous Hulthén potential $[37,38]$;

$$
\Delta(r)=-\Delta_{0} \frac{e^{-\delta r}}{1-e^{-\delta r}}
$$

where $\delta$ is the screening parameter which is used for determining the range of the Hulthén potential. The parameter $\Delta_{0}$ represents $\delta Z e^{2}$, where $Z e$ is the charge of the nucleon [39]. The intensity of the Hulthén potential is denoted by $\Delta_{0}$ under the condition of $\delta>0$. This potential has been used in several branches of physics and its discrete and continuum states have been studied by a variety of techniques such as the algebraic perturbation calculations which are based upon the dynamical group structure $\mathrm{SO}(2,1)$ [40], the formalism of supersymmetric quantum mechanics within the framework of the variational method [41], the supersymmetry and shape invariance property [42], the asymptotic iteration method [43,44] and the approach proposed by Biedenharn for the Dirac-Coulomb problem $[45,46]$. With this potential in place, Eq.(32) has to be solved numerically because the exponential behavior of $\Delta(r)$ is not compatible with the quadratic behavior of the pseudo-centrifugal kinetic energy term. However, Eq.(32) is analytically solvable only for the zero value of the pseudo-orbital angular momentum, i.e., $\tilde{\ell}=0(\kappa=1)$. In order to obtain more realistic results relating to the degenerate states, the Dirac equation should be solved for any $\tilde{\ell}$-states. In one of the methods used for solving Eq.(32), Hulthén square approximation can be introduced as an effective approximation to the pseudo-centrifugal kinetic energy term in the case of $\tilde{\ell}>0$ and small $r$. Following the original work of Filho et al [41] for this approximation, an effective potential term can be considered as follows

$$
\frac{\tilde{\ell}(\tilde{\ell}+1) \delta^{2} e^{-2 \delta r}}{\left(1-e^{-\delta r}\right)^{2}}=\frac{\tilde{\ell}(\tilde{\ell}+1) \delta^{2}}{([1+\delta r+\ldots]-1)^{2}} \simeq \frac{\tilde{\ell}(\tilde{\ell}+1)}{r^{2}} .
$$

The exponential numerator in Eq.(34) is expanded for small values of $r$ and higher-order

terms are ignored up to first-order term. Recently, the authors of $[42,44,46]$ have been used a more efficient approximation than that of Eq.(34) instead of the pseudo-centrifugal kinetic 
energy term $\tilde{\ell}(\tilde{\ell}+1) / r^{2}$. This approximation has the advantage that it is only valid for small values of $\delta$ and $\tilde{\ell}$. Whereas the present approximation in Eq.(34) can also be used for small values of $\delta$ and $\tilde{\ell}$..

When $\Delta(r)$ is taken as the Hulthén potential and the approximation of the centrifugal term as in Eq.(34), Eq.(32) yields

$$
\left[\frac{d^{2}}{d r^{2}}-\frac{\tilde{\ell}(\tilde{\ell}+1) \delta^{2} e^{-2 \delta r}}{\left(1-e^{-\delta r}\right)^{2}}-\left(\mu-E_{n_{r} \kappa}+C\right) \frac{\Delta_{0} e^{-\delta r}}{1-e^{-\delta r}}+E_{n_{r} \kappa}^{2}-\mu^{2}-C\left(\mu+E_{n_{r} \kappa}\right)\right] G_{n_{r} \kappa}(r)=0,
$$

where $\kappa=\tilde{\ell}+1$ for $\kappa>0$ and $\kappa=-\tilde{\ell}$ for $\kappa<0$ and the wave function has to satisfy the boundary conditions, i.e., $G_{n_{r} \kappa}(r=0)=0$ and $G_{n_{r} \kappa}(r \rightarrow \infty)=0$. It is convenient to introduce the following variable and parameters:

$$
\begin{gathered}
s=e^{-\delta r}, r \in(0, \infty) \rightarrow \mathrm{s} \in[0,1] \\
\nu_{1}^{2}=\frac{\left(\mu-E_{n_{r} \kappa}+C\right) \Delta_{0}}{\delta^{2}} \\
\omega_{1}^{2}=\frac{E_{n_{r} \kappa}^{2}-\mu^{2}-C \mu-C E_{n_{r} \kappa}}{\delta^{2}}, \\
A_{1}=\omega_{1}^{2}+\nu_{1}^{2}-\tilde{\ell}(\tilde{\ell}+1), \\
B_{1}=2 \omega_{1}^{2}+\nu_{1}^{2},
\end{gathered}
$$

which allows us to rewrite Eq.(35) in the simple form

$$
\left(\frac{d^{2}}{d s^{2}}+\frac{1-s}{s(1-s)} \frac{d}{d s}+\frac{A_{1} s^{2}-B_{1} s+\omega_{1}^{2}}{s^{2}(1-s)^{2}}\right) G_{n_{r} \kappa}(s)=0
$$

where the finiteness of our solution requires that $G_{n_{r} \kappa}(s=1)=0$ for $r \rightarrow 0$ and $G_{n_{r} \kappa}(s=$ $0)=0$ for $r \rightarrow \infty$. The above equation can be solved by using a special solution method mentioned in Ref.[27] and following a short-cut procedure given in Section 2. First of all, before starting the procedure of the solution, Eq.(41) is compared with the hypergeometric type differential equation given in Eq.(1) and consequently Eq.(41) is solved analytically due to the fact that the solution is still subjected to a methodology using algebra and calculus. This part will be treated in the next subsection partially.

\section{B. Pseudospin Symmetry Solution}

By applying the basic ideas of Ref.[27] and imposing the theory of orthogonal functions which are known as a generalization of the Rodrigues formula [35], the comparison of the 
differential equations in Eq.(41) and Eq.(1) gives us the following polynomials;

$$
\widetilde{\tau}(s)=1-s, \quad \sigma(s)=s(1-s), \quad \widetilde{\sigma}(s)=A_{1} s^{2}-B_{1} s+\omega_{1}^{2} .
$$

In the present case, if we want to substitute the polynomials given by Eq.(42) into Eq.(15), the following equality for the polynomial $\pi(s)$ is obtained

$$
\pi(s)=-\frac{s}{2} \pm \frac{1}{2} \sqrt{\left(1-4 A_{1}-4 k\right) s^{2}+4\left(B_{1}+k\right) s-4 \omega_{1}^{2}} .
$$

The expression under the square root of the above equation must be the square of a polynomial of first degree. This is possible only if its discriminant is zero and the constant parameter $k$ can be determined from the condition that the expression under the square root has a double zero. Hence, $k$ is obtained as $k_{+,-}=2 \omega_{1}^{2}-B_{1} \pm i \omega_{1}(2 \tilde{\ell}+1)$. In that case, it can be written in the four possible forms of $\pi(s)$;

$$
\left\{\begin{array}{l}
\pi(s)=-\frac{s}{2} \pm \frac{1}{2}\left(-\left[2 \tilde{\ell}+1-2 i \omega_{1}\right] s-2 i \omega_{1}\right), \text { for } k_{+}=2 \omega_{1}^{2}-B_{1}+i \omega_{1}(2 \tilde{\ell}+1) \\
\pi(s)=-\frac{s}{2} \pm \frac{1}{2}\left(-\left[2 \tilde{\ell}+1+2 i \omega_{1}\right] s+2 i \omega_{1}\right), \text { for } k_{-}=2 \omega_{1}^{2}-B_{1}-i \omega_{1}(2 \tilde{\ell}+1)
\end{array}\right.
$$

One of the four possible forms of $\pi(s)$ must be chosen to obtain an eigenvalue equation. Therefore, its most suitable form can be established by

$$
\pi(s)=i \omega_{1}-\left(i \omega_{1}+\tilde{\ell}+1\right) s
$$

for $k_{-}$. The trick in this selection is to find the negative derivative of $\tau(s)$ given in Eq.(6). Hence, $\tau(s)$ and $\tau^{\prime}(s)$ are obtained as

$$
\tau(s)=1+2 i \omega_{1}-\left(2 i \omega_{1}+2 \tilde{\ell}+3\right) s, \tau^{\prime}(s)=-\left(2 i \omega_{1}+2 \tilde{\ell}+3\right)<0 .
$$

In this case, a new eigenvalue equation for the Dirac equation becomes

$$
\lambda_{n_{r}}=n_{r}^{2}+2 n_{r}(\tilde{\ell}+1)+2 n_{r} i \omega_{1}
$$

where it is beneficial to invite the quantity $\lambda_{n_{r}}=-n_{r} \tau^{\prime}(s)-\frac{n_{r}\left(n_{r}-1\right)}{2} \sigma^{\prime \prime}(s)$ in Eq.(23). An other eigenvalue equation is obtained from the equality $\lambda=k_{-}+\pi^{\prime}$ in Eq.(14),

$$
\lambda=-\nu^{2}-(\tilde{\ell}+1)(1+2 i \omega) .
$$


In order to find an eigenvalue equation, the right-hand sides of Eq.(46) and Eq.(47) must be compared with each other. In this case the result obtained will depend on $E_{n_{r} \kappa}$ in the closed form:

$$
-\omega_{1}^{2}=\left(\frac{\left(1+2 n_{r}\right)(\tilde{\ell}+1)+n_{r}^{2}+\nu_{1}^{2}}{2\left(n_{r}+\tilde{\ell}+1\right)}\right)^{2} .
$$

Substituting the terms of right-hand sides of Eqs.(37) and (38) into Eq.(48), the energy eigenvalue equation for $E_{n_{r} \kappa}$ can be immediately obtained;

$$
\left(1+\left(\frac{\Delta_{0}}{Y \delta}\right)^{2}\right) E_{n_{r} \kappa}^{2}-\left(C+\frac{2 T \Delta_{0}}{Y^{2}}\right) E_{n_{r} \kappa}+\left(\frac{T \delta}{Y}\right)^{2}-\mu^{2}-C \mu=0,
$$

where

$$
\begin{gathered}
U=\left(1+2 n_{r}\right)(\tilde{\ell}+1)+n_{r}^{2}, \\
Y=2\left(n_{r}+\tilde{\ell}+1\right), \\
T=U+\frac{(C+\mu) \Delta_{0}}{\delta^{2}} .
\end{gathered}
$$

The energy spectrum of the Dirac equation for $\Delta(r)=V(r)-S(r)=-\Delta_{0} \frac{e^{-\delta r}}{1-e^{-\delta r}}$ is obtained by means of Eq.(49). In this case, the states with the same $n_{r}$ and $\tilde{\ell}$ will be degenerate. The two energy solutions of the quadratic equation can be obtained as

$$
E_{n_{r} \kappa}^{ \pm}=\frac{\delta^{2}\left(2 \Delta_{0} T+C Y^{2}\right) \pm \sqrt{4 \delta^{2}\left(\Delta_{0}(C+\mu)-\delta^{2} T\right)\left(\Delta_{0} \mu+\delta^{2} T\right) Y^{2}+\delta^{4}(C+2 \mu)^{2} Y^{4}}}{2\left(\Delta_{0}^{2}+Y^{2} \delta^{2}\right)} .
$$

For a given value of $n_{r}$ and $\kappa$ (or $\widetilde{\ell}$ ), the above equation provides two distinct positive and negative energy spectra related with $E_{n_{r} \kappa}^{+}$or $E_{n_{r} \kappa}^{-}$, respectively. One of the distinct solutions is only valid to obtain the negative-energy bound states in the limit of the pseudospin symmetry. Before seeking the acceptable solution, it is useful to present some analogy about the energy spectra.

Now, we are going to find the corresponding wave functions for the present potential model. Firstly, we calculate the weight function defined as [47-51]

$$
\rho(s)=\frac{1}{\sigma(s)} \exp \left(\int \frac{\tau(s)}{\sigma(s)} d s\right)=s^{2 i \omega_{1}}(1-s)^{2 \tilde{\ell}+1},
$$

and the first part of the wave function in Eq. (4):

$$
\phi(s)=\exp \left(\int \frac{\pi(s)}{\sigma(s)} d s\right)=s^{i \omega_{1}}(1-s)^{\tilde{\ell}+1} .
$$


Hence, the second part of the wave function which is the solution of Eq.(20) can be obtained by means of the so called Rodrigues representation

$$
\begin{gathered}
y_{n_{r}}(s)=c_{n_{r} \kappa} s^{-2 i \omega_{1}}(1-s)^{-(2 \tilde{\ell}+1)} \frac{d^{n_{r}}}{d s^{n_{r}}}\left[s^{n_{r}+2 i \omega_{1}}(1-s)^{n_{r}+2 \tilde{\ell}+1}\right] \\
\sim P_{n_{r}}^{\left(2 i \omega_{1}, 2 \tilde{\ell}+1\right)}(1-2 s), s \in[0,1],
\end{gathered}
$$

where the Jacobi polynomial $P_{n_{r}}^{(\mu, \nu)}(x)$ is defined for $\operatorname{Re}(\nu)>-1$ and $\operatorname{Re}(\mu)>-1$ for the argument $x \in[-1,+1]$ and $c_{n_{r} \kappa}$ is the normalization constant. By using $G_{n_{r} \kappa}(s)=$ $\phi(s) y_{n_{r}}(s)$, in this way we may write the lower-spinor wave function in the following fashion

$$
\begin{aligned}
G_{n_{r} \kappa}(r)= & c_{n_{r} \kappa}\left(\exp \left(-i \omega_{1} \delta r\right)\right)(1-\exp (-\delta r))^{\tilde{\ell}+1} P_{n}^{\left(2 i \omega_{1}, 2 \tilde{\ell}+1\right)}(1-2 \exp (-\delta r)) \\
& =c_{n_{r} \kappa} \frac{\left(2 i \omega_{1}+1\right)_{n_{r}}}{n_{r} !}\left(\exp \left(-i \omega_{1} \delta r\right)\right)(1-\exp (-\delta r))^{\tilde{\ell}+1} \\
& \times{ }_{2} F_{1}\left(-n_{r}, n_{r}+2\left(i \omega_{1}+\tilde{\ell}+1\right) ; 1+2 i \omega_{1} ; \exp (-\delta r)\right),
\end{aligned}
$$

where

$$
i \omega_{1} \delta=\sqrt{C\left(\mu+E_{n_{r} \kappa}\right)+\mu^{2}-E_{n_{r} \kappa}^{2}}>0 .
$$

The hypergeometric series ${ }_{2} F_{1}\left(-n_{r}, n_{r}+2\left(i \omega_{1}+\tilde{\ell}+1\right) ; 1+2 i \omega_{1} ; \exp (-\delta r)\right)$ terminates for $n_{r}=0$ and thus converges for all values of real parameters $\omega_{1}>0$ and $\tilde{\ell}>0$. In case if $C=0$, then $i \omega_{1} \delta=\sqrt{\left(\mu+E_{n_{r} \kappa}\right)\left(\mu-E_{n_{r} \kappa}\right)}$ with the following restriction $E_{n_{r} \kappa}<\mu$ required to obtain bound state (real) solutions for both positive and negative solutions of $E_{n_{r} \kappa}$ in Eq. (53). Now, before presenting the corresponding upper-component $F_{n_{r} \kappa}(r)$, let us recall a recurrence relation of hypergeometric function

$$
\frac{d}{d s}\left[{ }_{2} F_{1}(a ; b ; c ; s)\right]=\left(\frac{a b}{c}\right){ }_{2} F_{1}(a+1 ; b+1 ; c+1 ; s),
$$

with which the corresponding upper component $F_{n_{r} \kappa}(r)$ can be given by solving Eq. (28) as follows

$$
\begin{aligned}
F_{n_{r} \kappa}(r)=d_{n_{r} \kappa} & \frac{\left(\exp \left(-i \omega_{1} \delta r\right)\right)(1-\exp (-\delta r))^{\tilde{\ell}+1}}{\left(\mu-E_{n_{r} \kappa}+C\right)}\left[\frac{(\tilde{\ell}+1) \delta \exp (-\delta r)}{(1-\exp (-\delta r))}-i \omega_{1} \delta-\frac{\kappa}{r}\right] \\
& \times{ }_{2} F_{1}\left(-n_{r}, n_{r}+2\left(i \omega_{1}+\tilde{\ell}+1\right) ; 1+2 i \omega_{1} ; \exp (-\delta r)\right) \\
+d_{n_{r} \kappa} & {\left[\frac{n_{r}\left[n_{r}+2\left(i \omega_{1}+\tilde{\ell}+1\right)\right] \delta(\exp (-\delta r))^{i \omega_{1}+1}(1-\exp (-\delta r))^{\tilde{\ell}+1}}{\left(1+2 i \omega_{1}\right)\left(\mu-E_{n_{r} \kappa}+C\right)}\right] }
\end{aligned}
$$




$$
\times{ }_{2} F_{1}\left(1-n_{r}, n_{r}+2\left(i \omega_{1}+\tilde{\ell}+\frac{3}{2}\right) ; 2\left(1+i \omega_{1}\right) ; \exp (-\delta r)\right),
$$

where $E_{n_{r} \kappa} \neq \mu$ when $C=0$, exact pseudospin symmetry and $d_{n_{r} \kappa}$ is the normalization factor.

\section{Spin Symmetry Solution}

This symmetry arises from the near equality in magnitude of an attractive scalar, $S(\vec{r})$, and repulsive vector, $V(\vec{r})$, relativistic mean field, $S(\vec{r}) \sim V(\vec{r})$ in which the nucleon move [48-51]. Therefore, we simply take the sum potential equal to the isotonic potential model, i.e.,

$$
\Sigma(r)=-\Sigma_{0} \frac{e^{-\delta r}}{1-e^{-\delta r}}
$$

along with the approximation given by Eq.(34) to deal with the spin-orbit centrifugal term $\ell(\ell+1) / r^{2}$. In the last equation, the choice of $\Sigma(r)=2 V(r) \rightarrow V(r)$ allows us to reduce the

resulting solutions of the Dirac equation into their non-relativistic limits under appropriate choice of parameter transformations [51]. Therefore, the spin-symmetry Dirac equation (31) becomes

$$
\left\{\frac{d^{2}}{d r^{2}}-\frac{\ell(\ell+1) \delta^{2} e^{-2 \delta r}}{\left(1-e^{-\delta r}\right)^{2}}+\left(\mu+E_{n_{r} \kappa}-C\right) \frac{\Sigma_{0} e^{-\delta r}}{1-e^{-\delta r}}-\left[\mu^{2}-E_{n_{r} \kappa}^{2}-C\left(\mu-E_{n_{r} \kappa}\right)\right]\right\} F_{n_{r} \kappa}(r)=0,
$$

where $\kappa=\ell$ for $\kappa>0$ and $\kappa=-(\ell+1)$ for $\kappa<0$. It is convenient to introduce the following new variable and parameters:

$$
\begin{gathered}
s=e^{-\delta r}, r \in(0, \infty) \rightarrow \mathrm{s} \in[0,1] \\
\nu_{2}^{2}=\frac{\left(\mu+E_{n_{r} \kappa}-C\right) \Sigma_{0}}{\delta^{2}}, \\
\omega_{2}^{2}=\frac{E_{n_{r} \kappa}^{2}-\mu^{2}+C \mu-C E_{n_{r} \kappa}}{\delta^{2}}, \\
A_{2}=\omega_{2}^{2}-\nu_{2}^{2}-\ell(\ell+1), \\
B_{2}=2 \omega_{2}^{2}-\nu_{2}^{2},
\end{gathered}
$$

which allow us to rewrite Eq.(62) in a more simple form as

$$
\left(\frac{d^{2}}{d s^{2}}+\frac{1-s}{s(1-s)} \frac{d}{d s}+\frac{A_{2} s^{2}-B_{2} s+\omega_{2}^{2}}{s^{2}(1-s)^{2}}\right) F_{n_{r} \kappa}(s)=0
$$


where the finiteness of our solutions require that $F_{n_{r} \kappa}(1)=0$ and $F_{n_{r} \kappa}(0) \rightarrow 0$. We apply the NU method following the same steps of solution in previous section to obtain the expressions:

$$
\widetilde{\tau}(s)=1-s, \sigma(s)=s(1-s), \tilde{\sigma}(s)=A_{2} s^{2}-B_{2} s+\omega_{2}^{2} .
$$

To avoid repition, the functions required by the method for $\pi(s), k$ and $\tau(s)$ can be established as

$$
\begin{aligned}
& \pi(s)=i \omega_{2}-\left(i \omega_{2}+\ell+1\right) s, \\
& k=2 \omega_{2}^{2}-B_{2}-i \omega_{2}(2 \ell+1),
\end{aligned}
$$

and

$$
\tau(s)=1+2 i \omega_{2}-\left(2 i \omega_{2}+2 \ell+3\right) s, \tau^{\prime}(s)=-\left(2 i \omega_{2}+2 \ell+3\right)<0 .
$$

respectively, with prime denotes the derivative with respect to $s$. Also, the parameters $\lambda$ and $\lambda_{n}$ take the forms:

$$
\lambda_{n_{r}}=n_{r}^{2}+2 n_{r}(\ell+1)+2 n_{r} i \omega_{2} \text { and } \lambda=\nu_{2}^{2}-(\ell+1)\left(1+2 i \omega_{2}\right) .
$$

Using the basic condition $\lambda=\lambda_{n}$ followed by simple algebra, we obtain

$$
-\omega_{2}^{2}=\left(\frac{\left(1+2 n_{r}\right)(\ell+1)+n_{r}^{2}-\nu_{2}^{2}}{2\left(n_{r}+\ell+1\right)}\right)^{2}, n_{r}, \ell=1,2,3, \cdots
$$

and then the energy eigenvalue equation is immediately obtained

$$
\left(1+\left(\frac{\Sigma_{0}}{Z \delta}\right)^{2}\right) E_{n_{r} \kappa}^{2}-\left(C+\frac{2 S \Sigma_{0}}{Z^{2}}\right) E_{n_{r} \kappa}+\left(\frac{S \delta}{Z}\right)^{2}-\mu^{2}+C \mu=0,
$$

where

$$
\begin{gathered}
W=\left(1+2 n_{r}\right)(\ell+1)+n_{r}^{2}, \\
Z=2\left(n_{r}+\ell+1\right), \\
S=W+\frac{(C-\mu) \Sigma_{0}}{\delta^{2}} .
\end{gathered}
$$

The two energy solutions of the quadratic equation (75) can be obtained as

$$
E_{n_{r} \kappa}^{ \pm}=\frac{\delta^{2}\left(2 \Sigma_{0} S+C Z^{2}\right) \pm \sqrt{4 \delta^{2}\left(\Sigma_{0}(C-\mu)-\delta^{2} S\right)\left(-\Sigma_{0} \mu+\delta^{2} S\right) Z^{2}+\delta^{4}(C-2 \mu)^{2} Z^{4}}}{2\left(\Sigma_{0}^{2}+Z^{2} \delta^{2}\right)}
$$


For a given value of $n_{r}$ and $\kappa$ (or $\ell$ ), the above equation provides two distinct positive and negative energy spectra related with $E_{n_{r} \kappa}^{+}$or $E_{n_{r} \kappa}^{-}$, respectively. One of the distinct solutions is only valid to obtain the positive-energy bound states in the limit of the spin symmetry.

In our calculations for the spin symmetry wave functions, we firstly find the weight function:

$$
\rho(s)=s^{2 i \omega_{2}}(1-s)^{2 \ell+1}
$$

and from which the second part of the wave function by means of Rodrigues formula as

$$
\begin{gathered}
y_{n_{r}}(s)=a_{n_{r} \kappa} s^{-2 i \omega_{2}}(1-s)^{-(2 \ell+1)} \frac{d^{n_{r}}}{d s^{n_{r}}}\left[s^{n_{r}+2 i \omega_{2}}(1-s)^{n_{r}+2 \ell+1}\right] \\
\sim P_{n_{r}}^{\left(2 i \omega_{2}, 2 \ell+1\right)}(1-2 s), s \in[0,1],
\end{gathered}
$$

where the Jacobi polynomial $P_{n_{r}}^{(\mu, \nu)}(x)$ is defined for $\operatorname{Re}(\nu)>-1$ and $\operatorname{Re}(\mu)>-1$ for the argument $x \in[-1,+1]$ and $a_{n_{r} \kappa}$ is the normalization constant. Further, the first part of the wave function is being calculated as

$$
\phi(s)=s^{i \omega_{2}}(1-s)^{\ell+1} .
$$

By using $F_{n_{r} \kappa}(s)=\phi(s) y_{n_{r}}(s)$, in the spin symmetry case, we may write down the upperspinor wave function in the following fashion

$$
\begin{aligned}
F_{n_{r} \kappa}(r)= & a_{n_{r} \kappa}\left(\exp \left(-i \omega_{2} \delta r\right)\right)(1-\exp (-\delta r))^{\ell+1} P_{n_{r}}^{\left(2 i \omega_{2}, 2 \ell+1\right)}(1-2 \exp (-\delta r)) \\
= & a_{n_{r} \kappa} \frac{\left(2 i \omega_{2}+1\right)_{n_{r}}}{n_{r} !}\left(\exp \left(-i \omega_{2} \delta r\right)\right)(1-\exp (-\delta r))^{\ell+1} \\
& \times{ }_{2} F_{1}\left(-n_{r}, n_{r}+2\left(i \omega_{2}+\ell+1\right) ; 1+2 i \omega_{2} ; \exp (-\delta r)\right)
\end{aligned}
$$

where

$$
i \omega_{2} \delta=\sqrt{C\left(E_{n_{r} \kappa}-\mu\right)+\mu^{2}-E_{n_{r} \kappa}^{2}}>0 .
$$

It is noted that the hypergeometric series ${ }_{2} F_{1}\left(-n_{r}, n_{r}+2\left(i \omega_{2}+\ell+1\right) ; 1+2 i \omega_{2} ; \exp (-\delta r)\right)$ terminates for $n_{r}=0$ and thus it converges for all values of real parameters $\omega_{2}>0$ and $\ell>0$. In case when $C=0$, then $i \omega_{1} \delta=\sqrt{\left(\mu+E_{n_{r} \kappa}\right)\left(\mu-E_{n_{r} \kappa}\right)}$ with a restriction for real bound states that $E_{n_{r} \kappa}<\mu$ for both positive and negative solutions of $E_{n_{r} \kappa}$ in Eq. (79). Thus, the corresponding spin-symmetric lower-component $G_{n_{r} \kappa}(r)$ can be found as follows

$$
G_{n_{r} \kappa}(r)=b_{n_{r} \kappa} \frac{\left(\exp \left(-i \omega_{2} \delta r\right)\right)(1-\exp (-\delta r))^{\ell+1}}{\left(\mu+E_{n_{r} \kappa}-C\right)}\left[\frac{(\ell+1) \delta \exp (-\delta r)}{(1-\exp (-\delta r))}-i \omega_{2} \delta+\frac{\kappa}{r}\right]
$$




$$
\begin{gathered}
\times{ }_{2} F_{1}\left(-n_{r}, n_{r}+2\left(i \omega_{2}+\ell+1\right) ; 1+2 i \omega_{2} ; \exp (-\delta r)\right) \\
+b_{n_{r} \kappa}\left[\frac{n_{r} \delta\left[n_{r}+2\left(\ell+1+i \omega_{2}\right)\right](\exp (-\delta r))^{i \omega_{2}+1}(1-\exp (-\delta r))^{\ell+1}}{\left(1+2 i \omega_{1}\right)\left(\mu+E_{n_{r} \kappa}-C\right)}\right] \\
\times{ }_{2} F_{1}\left(1-n_{r}, n_{r}+2\left(i \omega_{2}+\ell+\frac{3}{2}\right) ; 2\left(1+i \omega_{2}\right) ; \exp (-\delta r)\right),
\end{gathered}
$$

where $E_{n_{r} \kappa} \neq-\mu$ when $C=0$, exact spin symmetry and $b_{n_{r} \kappa}$ is the normalization constant.

Let us finally remark that a careful inspection to our present spin-symmetric solution shows that it can can be easily recovered by knowing the relationship between the present set of parameters $\left(\omega_{2}^{2}, \nu_{2}^{2}, A_{2}, B_{2}\right)$ and the previous set of parameters $\left(\omega_{1}^{2}, \nu_{1}^{2}, A_{1}, B_{1}\right)$. This tells us that the positive energy solution for spin symmetry (negative energy solution for pseudospin symmetry) can be obtained directly from those of the negative energy solution for pseudospin symmetry (positive energy solution for spin symmetry) by performing the following replacements [48-51]:

$$
\begin{gathered}
F_{n_{r} \kappa}(r) \leftrightarrow G_{n_{r} \kappa}(r), V(r) \rightarrow-V(r)\left(\text { or } \Sigma_{0} \leftrightarrow-\Delta_{0}\right), \ell(\ell+1) \leftrightarrow \tilde{\ell}(\tilde{\ell}+1) \\
, E_{n_{r} \kappa}^{+} \leftrightarrow-E_{n_{r} \kappa}^{-}, \omega_{2}^{2} \leftrightarrow \omega_{1}^{2} \text { and } \nu_{2}^{2} \leftrightarrow-\nu_{1}^{2} .
\end{gathered}
$$

That is, with the above replacements, Eqs. (49) and (57) yield Eqs. (75) and (83) and vice versa is true.

Let us now present the non-relativistic limit. This can be achieved when we set $C=0$, $\Sigma_{0}=\delta$ and using the mapping $E_{n_{r} \kappa}-\mu \rightarrow E_{n_{r} \ell}$ and $E_{n_{r} \kappa}+\mu \rightarrow 2 \mu$ in Eqs.(64), (65) and (74), then the resulting energy eigenvalues (in $\hbar=c=e=1$ units) are

$$
E_{n_{r} \ell}=-\frac{1}{2 \mu}\left[\frac{\left(1+2 n_{r}\right)(\ell+1) \delta+n_{r}^{2} \delta-2 \mu}{2\left(n_{r}+\ell+1\right)}\right]^{2}, n_{r}, \ell=0,1,2,3, \cdots .
$$

Also, the wave functions in Eqs.(83) and (84) turns out to become

$$
\begin{gathered}
\left.R_{n_{r} \ell}(r)=a_{n_{r} \ell} r^{-1}\left(\exp \left(-\sqrt{-2 \mu E_{n_{r} \ell}} r\right)\right)(1-\exp (-\delta r))^{\ell+1} P_{n_{r}}^{\left(2 \sqrt{-2 \mu E_{n_{r} \ell}} / \delta, 2 \ell+1\right.}\right)(1-2 \exp (-\delta r)) \\
\left.=a_{n_{r} \ell} \frac{\left(2 \sqrt{-2 \mu E_{n_{r} \ell}} / \delta+1\right)}{n_{r} !}\right)_{n_{r}} r^{-1}\left(\exp \left(-\sqrt{-2 \mu E_{n_{r}} \ell} r\right)\right)(1-\exp (-\delta r))^{\ell+1} \\
\times{ }_{2} F_{1}\left(-n_{r}, n_{r}+2\left(\sqrt{-2 \mu E_{n_{r} \ell}} / \delta+\ell+1\right) ; 1+2 \sqrt{-2 \mu E_{n_{r} \ell}} / \delta ; \exp (-\delta r)\right), E_{n_{r} \ell}<0 .
\end{gathered}
$$




\section{RESULTS AND CONCLUSIONS}

In the present study, the Dirac equation for the Hulthén potential is approximately solved under the condition of the exact spin and pseudospin symmetry within the framework of the relativistic mean field theory. By using the basic ideas of the NU method, the energy eigenvalue expression for the arbitrary pseudo-orbital angular momentum $\tilde{\ell}$ is obtained approximately. The second-order differential equation given in Eq.(32) is solved by applying the Hulthén square approximation to deal with the pseudospin-orbit and spin-orbit cen-

trifugal and kinetic energy terms $\tilde{\ell}(\tilde{\ell}+1) / r^{2}$ and $\ell(\ell+1) / r^{2}$. The energy spectrum for any $\tilde{\ell}$ states is obtained analytically. Under the condition of the exact pseudospin and spin symmetry limitations, the energy relations in the Dirac equation with equal scalar and vector Hulthén potentials are recovered to see degenerate states.

The results obtained for this motivation show the orbital dependency of the Dirac equation for the Hulthén potential. Certainly, an analysis detailed by solving Dirac equation in relativistic mean field theories needs to use a very large scale $(\sim 660 \mathrm{MeV})$ comparing to the nuclear physics scale (few $\mathrm{MeV})$ ) in point of the intensity of potentials [6]. For this reason, the intensity of the potential, $\Delta_{0}$, used in Eq.(33) is considered as $3.4 \mathrm{fm}^{-1}$. The units of $\hbar=c=e=1$ are used throughout the present work for the sake of simplicity. Hence, the energy eigenvalue expression given in Eq.(53) can be simply discussed by using a set of physical parameter values. In the below explanations, although the energy spectrums can be calculated in dimensionless or arbitrary units, the calculations are preferably made in $f m^{-1}$ for the energy, mass, $C$ and intensity of the potential.

It is noted that the energy spectrum given by Eq.(53) indicates a family of the pseudospin symmetry Hulthén potential. Moreover, the analytical expression for Eq.(53) can be confronted with the results of [44] which is slightly in agreement with the result presented in Eq.(47) for the pseudospin symmetry solution. The results are only valid for small values of $\delta$ and $\tilde{\ell}$ (or $\kappa$ ). This spectrum changes with the relevant quantum numbers as well as the screening parameter $\delta$. The variation of the energy spectrums $\left(E_{n_{r} \kappa}^{+}\right.$and $\left.E_{n_{r} \kappa}^{-}\right)$according to the screening parameter $\delta$ is shown in Fig.1a and Fig.1b, with the choices of parameters $C=-4.9 \mathrm{fm}^{-1}$ and $\mu=5.0 \mathrm{fm}^{-1}$, which is in the range of nucleon mass value $(\sim 1$ $\mathrm{GeV}$ ). Figure 1a indicates the positive-energy bound states, i.e., $E_{n_{r} \kappa}^{+}$, while Fig.1b shows the negative-energy bound states, i.e., $E_{n_{r} \kappa}^{-}$. For a given value of $n_{r}$ and $\tilde{\ell}$, it is seen that an 
increment on $\delta$ leads to an increment on $E_{n_{r} \kappa}^{-}$along the negative-energy direction whereas the same increment on $\delta$ results with a reduction on $E_{n_{r} \kappa}^{+}$along the positive-energy direction. The results presented in Fig.1a show that the energy difference between the states is still small although the values of screening parameter $\delta$ increases. Figure $1 \mathrm{~b}$ has two interesting results: The first one indicates that the negative-energy bond states appear with the large values of $\delta$ and $\tilde{\ell}$. The reason of this aspect comes from the approximation mentioned in the previous sections. The second one belongs to the small values of $\delta$. For instance, $E_{n_{r} \kappa}^{-}$for $1 s_{1 / 2}, 1 d_{7 / 2}$ and $1 g_{9 / 2}$ is valid under the condition of $\delta \gtrsim 0.09, \delta \gtrsim 0.05$ and $\delta \gtrsim 0.03$, respectively. Therefore, $E_{n_{r} \kappa}^{-}$still represents the negative-energy bound states for small values of $\delta$ when $\tilde{\ell}$ increases. These results can be also expanded on the other states of the Hulthén potential with the pseudospin symmetry.

It is well-known that for the finite nuclei the constant $C$ is adjusted to zero because each potential goes to zero at large distances. If the difference between the scalar $S(r)$ and vector $V(r)$ potentials equals to a given constant $C$, this is equivalent to adding the relevant constant to the relativistic energy and mass. The constant for the energy is unimportant because it does not affect the energy difference. Whereas the variation of $C$ is equivalent to the variation of mass. This is more physically transparent for the pseudospin-orbit dependency of the Dirac equation. Moreover, in the case of the infinite nuclear matter, the constant $C$ could be non-zero. The energy spectrum versus the mass $\mu$ is plotted by setting $C=-4.9 \mathrm{fm}^{-1}$, as shown in Fig.2. The variation of the energy spectrum for $\tilde{\ell}=1, \tilde{\ell}=3$, $\tilde{\ell}=5$ and $\tilde{\ell}=7$ is presented by using $\delta=0.25$. The radial quantum number is fixed to $1\left(n_{r}=1\right)$. In Fig.2, the dashed (red lines, see colour online) and solid (blue lines, see colour online) lines represent $E_{n_{r} \kappa}^{+}$and $E_{n_{r} \kappa}^{-}$, respectively. According to Fig.2, there are two different regions of energy spectrum versus the mass. For $0<\mu \leq 2.3 \mathrm{fm}^{-1}$ the energy spectrum is in the negative region completely. With the $\tilde{\ell}$ increasing, $E_{n_{r} \kappa}^{+}$fan out along the positive part of the energy spectrum whereas $E_{n_{r} \kappa}^{-}$overlaps in going from $\tilde{\ell}=1$ to $\tilde{\ell}=7$. The case of $E_{n_{r} \kappa}^{-}$in the interval of $0<\mu \leq 2.3 \mathrm{fm}^{-1}$ represents the degenerate states in the different values of $\tilde{\ell}$ for a given value of $n_{r}$. A similar trend is seen when $\delta \geq 3.2 \mathrm{fm}^{-1}$ in the several values of $\tilde{\ell}$ for $E_{n_{r} \kappa}^{+}$by crossing the zero axis toward the positive direction of the energy spectrum. However, the numerical results of $E_{n_{r} \kappa}^{+}$over the axis are not relevant for the negative-energy bound states. Meanwhile, the results of $E_{n_{r} \kappa}^{-}$are also valid under the conditions of $\delta \geq 3.9,3.5,3.3,3.2 \mathrm{fm}^{-1}$ for $\tilde{\ell}=1,3,5,7$, respectively, but only 
relevant in the large values of $\tilde{\ell}$. Furthermore, the energy spectrum versus the constant $C$ is plotted by taking $\mu=5.0 \mathrm{fm}^{-1}$ and $\delta=0.25$ as shown in Fig.3. According to Fig.3, it is seen that the negative values of $C$ show more strongly binding energies under the condition of $C \leq-11.0 \mathrm{fm}^{-1}$ for $E_{n_{r} \kappa}^{+}$(dashed lines) and $E_{n_{r} \kappa}^{-}$(blue line) in the whole values of $\tilde{\ell}$. Moreover, $E_{n_{r} \kappa}^{+}$(dashed line) still shows the negative-energy bound states on condition that $-8<C<-5 \mathrm{fm}^{-1}$ up to the zero axis. In the case of $E_{n_{r} \kappa}^{-}$(blue lines) the situation becomes different than that of $E_{n_{r} \kappa}^{+}$. The axis is crossed with changing $\tilde{\ell}$ for $E_{n_{r} \kappa}^{-}$. The crossing points of axis from $C=-8 \mathrm{fm}^{-1}$ become large with the $\tilde{\ell}$ increasing. In Table 1 , the degenerate states are presented for a Dirac particle within the Hulthén potential, with $C=-4.9 \mathrm{fm}^{-1}, \mu=5.0 \mathrm{fm}^{-1}$ and $\Delta_{0}=3.4 \mathrm{fm}^{-1}$. The several pseudo-orbital and radial quantum numbers are used in the numerical calculations to predict the orbital dependency of the Dirac equation under the condition of the exact pseudospin symmetry. As an example, the Dirac eigenstate $1 s_{1 / 2}$ with $n_{r}=1$ and $\kappa=-1$ will have a partner which is denoted by the $0 d_{3 / 2}$ with $n_{r}-1=0$ and $\kappa=2$. These states are called the pseudospin partner and degenerated with each other.

\section{Acknowledgments}

The partial support provided by the Scientific and Technical Research Council of Turkey (TÜBITAK) is highly appreciated. One of the authors (C.B.) acknowledges the financial support provided by the Science Foundation of Erciyes University. 
[1] A. Arima, M. Harvey, K. Shimizu, Pseudo LS coupling and pseudo SU3 coupling schemes, Phys. Lett. B 30 (1969) 517-522.

[2] K.T. Hecht, A. Adler, Generalized seniority for favored $J \neq 0$ pair in mixed configurations, Nucl. Phys. A 137 (1969) 129-143.

[3] J.N. Ginocchio, A relativistic symmetry in nuclei, Phys. Reports 315 (1999) 231-240.

[4] A. Leviatan, Supersymmetric Patterns in the Pseudospin, Spin, and Coulomb Limits of the Dirac Equation with Scalar and Vector Potentials, Phys. Rev. Lett. 92 (2004) 202501.

[5] J.N. Ginocchio, Pseudospin as a relativistic symmetry, Phys. Rev. Lett. 78 (3) (1997) 436-439.

[6] J.N. Ginocchio, Relativistic symmetries in nuclei and hadrons, Phys. Rep. 414 (4-5) (2005) 165-261.

[7] J. Meng, K. Sugawara-Tanabe, S. Yamaji, P. Ring, A. Arima, Pseudospin symmetry in relativistic mean field theory, Phys. Rev. C 58 (2) (1998) R628-R631.

[8] R. Lisboa, M. Malheiro, A.S. de Castro, P. Alberto and M. Fiolhais, Pseudospin symmetry and the relativistic harmonic oscillator, Phys. Rev. C 69 (2004) 024319. 15pp.

[9] P. Alberto, M. Fiolhais, M. Malheiro, A. Delfino, M. Chiapparini, Isospin Asymmetry in the Pseudospin Dynamical Symmetry, Phys. Rev. Lett. 86 (2001) 5015-5018.

[10] P. Alberto, M. Fiolhais, M. Malheiro, A. Delfino, M. Chiapparini, Pseudospin symmetry as a relativistic dynamical symmetry in the nucleus, Phys. Rev. C 65 (2002) 034307. 9pp.

[11] C. Ti-Sheng, L. Hong-Feng, M. Jie, Z. Shuang-Quan and Z. Shan-Gui, Pseudospin Symmetry in Relativistic Framework with Harmonic Oscillator Potential and Woods-Saxon Potential, Chin. Phys. Lett. 20 (2003) 358-361.

[12] J.Y. Guo, X.Z. Fang, X.F Xu, Pseudospin symmetry in the relativistic harmonic oscillator, Nucl. Phys. A 757 (2005) 411-421.

[13] Q. Xu and S.J. Zhu, Pseudospin symmetry and spin symmetry in the relativistic Woods-Saxon, Nucl. Phys. A 768 (2006) 161-169.

[14] C. Berkdemir, A. Berkdemir and R. Sever, Polynomial solutions of the Schrödinger equation for the generalized Woods-Saxon potential, Phys. Rev. C 72 (2005) 027001. 4pp. [Editorial Note, 74 (2006) 039902(E)].

[15] J.Y. Guo, J.C. Han and R.D. Wang, Pseudospin symmetry and the relativistic ring-shaped 
non-spherical harmonic oscillator, Phys. Lett. A 353 (2006) 378-382.

[16] C. Berkdemir, A. Berkdemir and R. Sever, Systematical approach to the exact solution of the Dirac equation for a deformed form of the Woods-Saxon potential, J. Phys. A: Math. Gen. 39 (2006) 13455-13464.

[17] C. Berkdemir, Pseudospin symmetry in the relativistic Morse potential including the spin-orbit coupling term, Nucl. Phys. A 770 (2006) 32-39.

[18] Y. Xu, S. He and C.-S. Jia, Approximate analytical solutions of the Dirac equation with the Pöschl-Teller potential including the spin-orbit coupling term, J. Phys. A: Math. Theor. 41 (2008) 255302-255309.

[19] C.S. Jia, P. Guo and X.L. Peng, Exact solution of the Dirac-Eckart problem with spin and pseudospin symmetry, J. Phys. A: Math. Theor. 39 (2006) 7737-7744.

[20] C.S. Jia, J.Y. Wang, S. He and L.T. Sun, Shape invariance and the supersymmetry WKB approximation for a diatomic molecule potential, J. Phys. A: Math. Gen. 33 (2000) 6993-6998.

[21] C.S. Jia, J.Y. Liu and P.Q. Wang, A new approximation scheme for the centrifugal term and the Hulthén potential, Phys. Lett. A 372 (2008) 4779-4782.

[22] C.S. Jia, Y.F. Diao, L.Z. Yi and T. Chen, Arbitrary l-wave solutions of the Schrödinger equation with the Hulthén potential, Int. J. Mod. Phys. A 24 (24) (2009) 4519-4528.

[23] C.S. Jia, T. Chen and L.G. Cui, Approximate analytical solutions of the Dirac equation with the generalized Pöschl-Teller potential including the pseudo-centrifugal term, Phys. Lett. A 373 (2009) 1621-1626.

[24] Y. Xu, S. He and C.S. Jia, Approximate analytical solutions of the Klein-Gordon equation with the Pöschl-Teller potential including the centrifugal term, Phys. Scr. 81 (2010) 045001.

[25] S.M. Ikhdair, On the bound-state solutions of the Manning-Rosen potential including an improved approximation to the orbital centrifugal term, Phys. Scr. 83 (2011) 015010.

[26] S.M. Ikhdair and J. Abu-Hasna, Quantization rule solution to the Hulthén potential in arbitrary dimension with a new approximate scheme for the centrifugal term, Phys. Scr. 83 (2011) 025002.

[27] A.F. Nikiforov, V.B. Uvarov, Special Functions of Mathematical Physics, Birkhauser, Basel, 1988.

[28] A. Berkdemir, C. Berkdemir and R. Sever, Eigenvalues and eigenfunctions of Woods-Saxon potential in PT-symmetric quantum mechanics, Mod. Phys. Lett. A 21 (2006) 2087-2097. 
[29] C. Berkdemir, Relativistic treatment of a spin-zero particle subject to a Kratzer-type potential, Am. J. Phys. 75 (2007) 81-86.

[30] Y.F. Cheng and T.Q Dai, Exact solution of the Schrödinger equation for the modified Kratzer potential plus a ring-shaped potential by the Nikiforov-Uvarov method, Phys. Scr. 75 (2007) 274-277.

[31] C. Berkdemir and R. Sever, Pseudospin symmetry solution of the Dirac equation with an angle-dependent potential, J. Phys. A: Math. Theor. 41 (2008) 045302. 11pp.

[32] S. Flügge, Practical Quantum Mechanics I and II, Springer-Verlang, Berlin, 1971.

[33] C. Berkdemir and J. Han, Any l-state solutions of the Morse potential through the Pekeris approximation and Nikiforov-Uvarov method, Chem. Phys. Lett. 409 (2005) 203-207.

[34] S. Haouat and L. Chetouani, Bound states of Dirac particle subjected to the pseudoscalar Hulthén potential, J. Phys. A: Math. Theor. 40 (34) (2007) 10541-10548.

[35] G. Szego, Orthogonal Polynomials, American Mathematical Society, New York, 1959.

[36] W. Greiner, B. Müller and J. Rafelski, Quantum Electrodynamics of Strong Fields: With an Introduction into Modern Relativistic Quantum Mechanics, Springer, 2nd edition, New York, 1985.

[37] C.S. Lai and W.C. Lin, Energies of the Hulthén potential for $l \neq 0$, Phys. Lett. A 78 (4) (1980) 335-337.

[38] Y.P. Varshni, Eigenenergies and oscillator strengths for the Hulthén potential, Phys. Rev A 41 (9) (1990) 4682-4689.

[39] U. Myhrman, A recurrence formula for obtaining certain matrix elements in the base of eigenfunctions of the Hamiltonian for a particular screened potential, J. Phys. A: Math. Gen. 16 (2) (1983) 263-270.

[40] B. Roy and R. Roychoudhury, Dirac equation with Hulthén potential: an algebraic approach, J. Phys. A: Math. Gen. 23 (21) (1990) 5095-5102.

[41] E.D. Filho and R.M. Ricotta, Supersymmetry, variational method and Hulthén potential, Mod. Phys. Lett. A 10 (1995) 1613-1618.

[42] S.W. Qian, B.W. Huang and Z.Y. Gu, Supersymmetry and shape invariance of the effective screened potential, New J. Phys. 4 (2002) 13.1.

[43] H. Ciftci, R. L. Hall, and N. Saad, Asymptotic iteration method for eigenvalue problems, J. Phys. A: Math. Gen. 36 (48) (2003) 11807. 10pp 
[44] A. Soylu, O. Bayrak and I. Boztosun, An approximate solution of Dirac-Hulthén problem with pseudospin and spin symmetry for any $\kappa$ state, J. Math. Phys. 48 (2007) 082302. 9 pp.

[45] L.C. Biedenharn, Remarks on the Relativistic Kepler Problem, Phys. Rev. 126 (1962) 845-851.

[46] S. Haouat and L. Chetouani, Approximate solutions of Klein-Gordon and Dirac equations in the presence of the Hulthén potential, Phys. Scr. 77 (2) (2008) 025005.

[47] S.M. Ikhdair, Rotation and vibration of diatomic molecule in the spatially-dependent mass Schrödinger equation with generalized q-deformed Morse potential, Chem. Phys. 361 (2009) $9-17$.

[48] S.M. Ikhdair and R. Sever, Solutions of the spatially-dependent mass Dirac equation with the spin and pseudospin symmetry for the Coulomb-like potential, Appl. Math. Comp. 216 (2) (2010) 545-555.

[49] S.M. Ikhdair and R. Sever, Approximate bound state solutions of Dirac equation with Hulthén potential including Coulomb-like tensor potential, Appl. Math. Comp. 216 (3) (2010) 911-923.

[50] S.M. Ikhdair, Approximate solutions of the Dirac equation for the Rosen-Morse potential including the spin-orbit centrifugal term, J. Math. Phys. 51 (2) (2010) 023525. 16pp.

[51] S.M. Ikhdair and R. Sever, Approximate analytical solutions of the generalized Woods-Saxon potentials including the spin-orbit coupling term and spin symmetry, Cent. Eur. J. Phys. 8 (4) (2010) 652-666. 
FIG. 1: The variation of the energy spectrum in units of $\mathrm{fm}^{-1}$ versus the screening parameter $\delta$.

FIG. 2: The variation of the energy spectrum versus the mass $\mu$. All parameters are in units of $f m^{-1}$.

FIG. 3: The variation of the energy spectrum versus the constant $C$. All parameters are in units of $\mathrm{fm}^{-1}$. 
TABLE I: The negative-energy degenerate states in units of $\mathrm{fm}^{-1}$ of the pseudospin-symmetry Hulthén potential for various values of $n_{r}, \tilde{\ell}$ and $\delta$. For a special case, $\mu=5 \mathrm{fm}^{-1}, \Delta_{0}=3.4 \mathrm{fm}^{-1}$ and $C=-4.9 \mathrm{fm}^{-1}$.

\begin{tabular}{|c|c|c|c|c|c|c|c|}
\hline$\tilde{\ell}$ & $n_{r}$ & Degenerate States & $E_{n_{r}, \kappa}$ & $\tilde{\ell}$ & $n_{r}$ & Degenerate States & $E_{n_{r}, \kappa}$ \\
\hline \multirow[t]{4}{*}{1} & 1 & $0.025\left(1 s_{1 / 2}, 0 d_{3 / 2}\right)$ & 0.0963638 & 1 & 2 & $0.025\left(2 s_{1 / 2}, 1 d_{3 / 2}\right)$ & 0.0928939 \\
\hline & & 0.100 & 0.0425738 & & & 0.100 & -0.0103694 \\
\hline & & 0.175 & -0.0710009 & & & 0.175 & -0.2174930 \\
\hline & & 0.250 & -0.2346580 & & & 0.250 & -0.4920870 \\
\hline \multirow[t]{4}{*}{2} & 1 & $0.025\left(1 p_{3 / 2}, 0 f_{5 / 2}\right)$ & 0.0912282 & 2 & 2 & $0.025\left(2 p_{3 / 2}, 1 f_{5 / 2}\right)$ & 0.0863238 \\
\hline & & 0.100 & -0.0363590 & & & 0.100 & -0.1078600 \\
\hline & & 0.175 & -0.2930130 & & & 0.175 & -0.4732160 \\
\hline & & 0.250 & -0.6351320 & & & 0.250 & -0.9131390 \\
\hline \multirow[t]{4}{*}{3} & 1 & $0.025\left(1 d_{5 / 2}, 0 g_{7 / 2}\right)$ & 0.0839128 & 3 & 2 & $0.025\left(2 d_{5 / 2}, 1 g_{7 / 2}\right)$ & 0.0775818 \\
\hline & & 0.100 & -0.1447100 & & & 0.100 & -0.2316110 \\
\hline & & 0.175 & -0.5760950 & & & 0.175 & -0.7705370 \\
\hline & & 0.250 & -1.0984500 & & & 0.250 & -1.3540100 \\
\hline \multirow[t]{4}{*}{4} & 1 & $0.025\left(1 f_{7 / 2}, 0 h_{9 / 2}\right)$ & 0.0744360 & 4 & 2 & $0.025\left(2 f_{7 / 2}, 1 h_{9 / 2}\right)$ & 0.0666955 \\
\hline & & 0.100 & -0.2784550 & & & 0.100 & -0.3771030 \\
\hline & & 0.175 & -0.8953110 & & & 0.175 & -1.0870200 \\
\hline & & 0.250 & -1.5671200 & & & 0.250 & -1.7758200 \\
\hline
\end{tabular}

